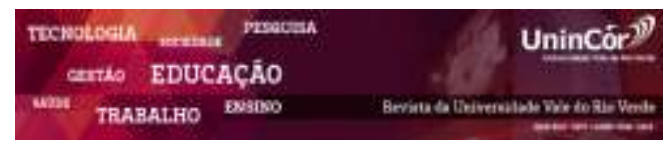

Revista da Universidade Vale do Rio Verde ISSN: 1517-0276 / EISSN: 2236-5362 Vol. 16 | n. 2 | Ano 2018

Vanessa Alves Mantovani Universidade Federal de Lavras (UFLA) vanismantovani@hotmail.com

Jhones da Silva Amorim Universidade Federal de Lavras (UFLA) jhonesamorim@gmail.com

André Ferreira Rodrigues Universidade Federal de Lavras (UFLA) afrodrigues09@gmail.com

Rubens Junqueira

Universidade Federal de Lavras (UFLA) rubensjunqueira@live.com

Ronan Naves Carvalho Universidade Federal de Lavras (UFLA) ronannaves@hotmail.com

Alisson Souza de Oliveira Universidade Vale do Rio Verde (UninCor) alissonso@hotmail.com

\section{ESTIMATIVAS DA TAXA DE ENTREGA DE SEDIMENTOS E ÁREAS SUSCEPÍTVEIS À EROSÃO EM UMA BACIA HIDROGRÁFICA NO SUL DE MINAS GERAIS - BRASIL}

\section{RESUMO}

O objetivo deste trabalho foi determinar a perda de solo e a taxa de entrega de sedimentos, bem como as áreas mais susceptíveis à erosão para o ano de 2016 em uma bacia hidrográfica situada no município de Lavras-MG. Para determinar a taxa de entrega de sedimentos foram aplicados os modelos RUSLE e MUSLE, sendo que para aplicação do segundo apenas a erosividade da chuva foi alterada, e calculado com base na Curva Número e Hidrograma Unitário Triangular. A taxa anual de perda de solo foi de 37,54 ton.ha ${ }^{-1}$, entretanto, $69 \%$ da bacia hidrográfica apresentou uma taxa anual de perda de solo baixa, de até $5 \mathrm{t} \mathrm{ha}^{-1}$. A perda de solo total obtida com a MUSLE foi de $0,518 \mathrm{t}^{-\mathrm{ha}^{-1}}$. ano ${ }^{-1}$, sendo que $66 \%$ foi gerado em apenas um evento. A taxa de entrega de sedimento anual na bacia hidrográfica foi de $1,38 \%$, valor este muito próximo ao encontrado na literatura para a mesma região. A aplicação dos modelos RUSLE e MUSLE mostrou-se adequada para estudo da taxa de entrega de sedimentos. Observou-se a necessidade da alteração do uso do solo e aplicação de práticas conservacionistas a fim de reduzir a perda de solo e a taxa de entrega de sedimentos.

Palavras-chave: Perda de solo. RUSLE. MUSLE. Conservação do solo. Escoamento superficial.

\section{ESTIMATES OF THE SEDIMENTS DELIVERY AND AREAS SUSCEPTIAL TO EROSION IN A HYDROGRAPHIC BASIN IN THE SOUTH OF MINAS GERAIS - BRAZIL}

\begin{abstract}
The objective of this work was to determine the soil loss and the sediment delivery ratio, as well as the most susceptible areas for erosion for the year 2016 in a river basin located in the municipality of Lavras-MG. To determine the sediment delivery ratio, the RUSLE and MUSLE models were applied, and for the second application only the rain erosivity was changed, and calculated based on the Number Curve and Triangular Unit Hydrograph. The annual rate of soil loss was 37.54 t.ha $^{-1}$, however, $69 \%$ of the river basin had an annual low soil loss rate of up to 5 ton.ha'. The total soil loss obtained with MUSLE was 0.518 t.ha ${ }^{-1} \cdot$ year $^{-1}$, with $66 \%$ being generated in only one event. The annual sediment delivery rate in the river basin was $1.38 \%$, which is very close to that found in the literature for the same
\end{abstract}


region. The application of the RUSLE and MUSLE models was adequate to study the sediment delivery ratio. It was observed the need of alteration of the use of the soil and application of conservationist practices in order to reduce the soil loss and the rate of sediment delivery.

Keywords: Soil loss. RUSLE. MUSLE. Soil conservation. Runoff.

\section{INTRODUÇÂO}

Os processos erosivos são um dos principais fatores que causam a deterioração dos solos ao redor do mundo. Por conseguinte, deve ser avaliado cuidadosamente, uma vez que afeta diretamente a capacidade produtiva dos solos e, consequentemente, a segurança alimentar mundial (RICKSON et al., 2015). Além do mais, Graves et al. (2015) estimaram que o custo anual decorrente da erosão do solo na Inglaterra e no País de Gales é de aproximadamente 150 milhões de libras. Logo, estudos que visem determinar seu comportamento e possíveis meios para contorná-lo são fundamentais.

Neste sentido vários modelos foram desenvolvidos no intuito de estimar as perdas de solo, sendo a equação universal de perda de solo USLE (WISCHMEIER; SMITH, 1965; 1978) pioneira quando se considera aplicação em âmbito mundial. Todavia, com o desenvolvimento tecnológico e as necessidades do conhecimento da erosão em escalas de bacias hidrográficas, surgiuse a forma revisada da equação universal de perda de solo - RUSLE (RENARD et al., 1991), a qual associada aos Sistemas de Informações Geográficas - SIG permite o conhecimento da erosão do solo de forma distribuída no espaço.
Porém, estes modelos estimam a perda de solo média anual de longo termo não sendo recomendáveis para eventos isolados. No entanto, tem sido utilizado equivocadamente para este devido fim (KINNELL, 2010). Para suprir essa demanda, modelos como a forma modificada da USLE/RUSLE conhecida como MUSLE equação modificada de perda de solo (KINNELL; RISSE, 1998) e aqueles mais baseados nos processos como o WEPP - WaterErosionPrediction Project (FLANAGAN; GILLEY; FRANTI, 2007) foram desenvolvidos.

Tais modelos têm sido amplamente utilizados na América do Sul (BONILLA; REYES; MAGRI, 2010; DELGADO; GASPARI; KRUSE, 2015), em especial no Brasil (SANTOS et al., 2014), para estimar as potenciais perdas de solo e as áreas de maiores riscos aos processos erosivos, além de auxiliar nas tomadas de decisões para a conservação e mitigação desses processos visando à sustentabilidade e a resiliência dos solos. Dessa forma, o presente trabalho tem como objetivo identificar as áreas mais susceptíveis à erosão, determinar a perda de solo anual aplicando os modelos MUSLE e RUSLE, bem como a taxa de entrega de sedimentos para o ano de 2016 em uma bacia hidrográfica urbana situada no município de Lavras-MG. 


\section{MATERIAL E MÉTODOS}

O trabalho foi conduzido em parte da bacia hidrográfica do Ribeirão Vermelho localizada no município de Lavras, sul de Minas Gerais, cuja área é de aproximadamente $13,38 \mathrm{~km}^{2}$ (Figura 1). Segundo Curi et al. (2015), o clima da região é do tipo Cwb, com verão chuvoso e inverno seco e frio de acordo com classificação de Köppen. De acordo com dados do Instituto Nacional de Meteorologia (INMET), a temperatura média anual do ar é em torno de $20,3^{\circ} \mathrm{C}$ e a precipitação média anual no munícipio é superior a $1460 \mathrm{~mm}$ por ano, porém nos últimos anos, devido às mudanças climáticas, a região tem enfrentado secas extremas.

Figura 1. Localização e Hidrografia da Bacia Hidrográfica analisada

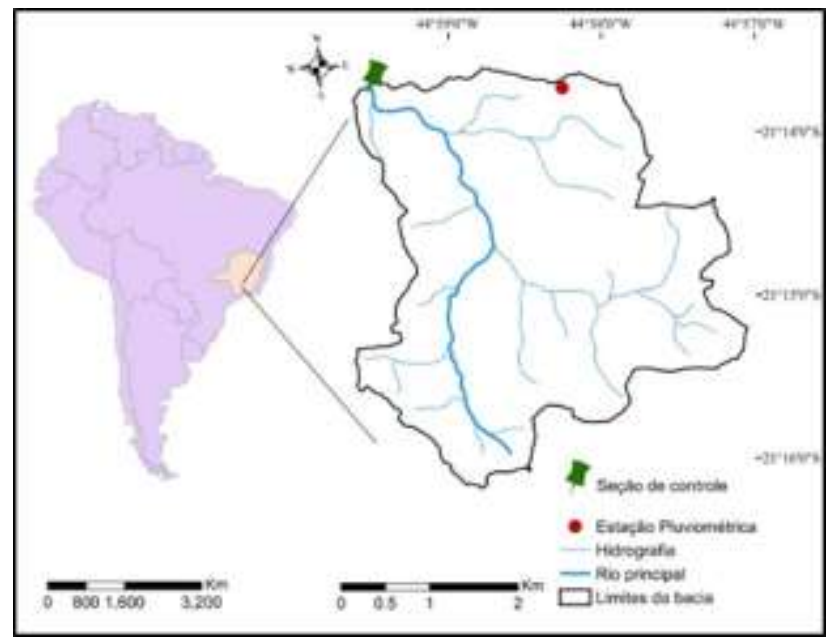

A bacia foi delimitada utilizando curvas de

nível, com 1 metro de resolução. A partir dessas curvas de nível foi gerado o Modelo Digital de Elevação (MDE) utilizando o software ArcGIS versão 9.3® e, posteriormente a delimitação da bacia com seção de controle nas coordenadas 2113’40,69”S; 4459’29,26”W. Já a hidrografia foi obtida junto ao banco de dados do Instituto Mineiro de Gestão das Águas.
Neste estudo, para calcular a taxa de perda de solo anual foi utilizada a equação da RUSLE, proposta por Renard et al. (1991). Esta equação inclui a análise de dados que não foram abordados anteriormente na USLE, além de uma atualização da teoria que descreve os processos hidrológicos e erosivos (PAN; WEN, 2014). A RUSLE é uma equação empírica (Equação 1) que quantifica a erosão média anual de uma bacia, geralmente expresso em t.ha ${ }^{-1} \cdot$ ano $^{-1}$.

$$
\mathrm{A}=\mathrm{R} * \mathrm{~K} * \mathrm{LS} * \mathrm{C} * \mathrm{P}
$$

em que: o fator $R$ se refere à erosividade da chuva, em MJ.mm.ha ${ }^{-1} \cdot$ ano $^{-1}$; o fator $K$ está associado à erodibilidade do solo, expresso em t.h. $\mathrm{MJ}^{-1} \cdot \mathrm{mm}^{-1}$; $L S$ está associado a topografia, adimensional; o fator $C$ corresponde ao manejo e cobertura do solo, adimensional e o fator $P$ se refere às práticas de conservação, adimensional.

$\mathrm{O}$ fator $\mathrm{R}$ foi obtido por meio dos dados mensais de chuva do ano de 2016 coletados na estação pluviométrica que se encontra no interior da bacia em estudo. Para seu cálculo, foi utilizada a equação desenvolvida por Aquino et al. (2012), a qual foi ajustada para o clima do município de Lavras.

$$
\mathrm{R}_{\mathrm{i}}=85,672 *\left(\frac{\mathrm{p}_{\mathrm{i}}^{2}}{\mathrm{~Pa}}\right)^{0,6557}
$$

em que: $R i$ representa a erosividade da chuva mensal, $p i$ a precipitação mensal e $P a$ a precipitação anual, sendo as duas últimas em $\mathrm{mm}$.

$\mathrm{O}$ fator $\mathrm{K}$ está relacionado com a erodibilidade do solo, ou seja, trata-se da vulnerabilidade do solo ou dos componentes superficiais em relação à erosão (KAYET et al., 2017). Para sua obtenção, foi feito o mapa de 
declividade da bacia através do MDE e, em seguida foi realizado a classificação do solo com base no mapa gerado. Após isso, foram atribuídos valores de $\mathrm{K}$ encontrados na literatura para cada tipo de solo. Vale ressaltar que áreas próximas aos cursos d'água foram consideradas como Gleissolo e Organossolo a um raio de $50 \mathrm{~m}$ do curso d'água principal e $30 \mathrm{~m}$ dos demais cursosd'água. Nas áreas onde existem dois tipos diferentes de solo, foram adotados valores médios de erodibilidade para as classes em questão.

O fator LS diz respeito ao comprimento do declive e à declividade. $\mathrm{O}$ comprimento do declive (L) está relacionado ao ponto no qual se inicia o escoamento superficial direto, até o ponto em que o declive diminui e começa a ocorrer deposição dos sedimentos erodidos. Este fator foi obtido por meio da ferramenta para SIG LS Tool desenvolvida por Zhang et al. (2013), onde é necessário entrar com o arquivo referente ao MDE.

O fator C está associado ao uso e ocupação do solo. Este fator pode ser utilizado para mostrar a influência de práticas de manejos na agriculturasobre a taxa de erosão do solo em terras agrícolas (KATHUN, 2017). O mapa de uso e ocupação do solo foi obtido por meio de classificação supervisionada por máxima verossimilhança utilizando imagens de satélite RapidEye 2014 com resolução de 5 metros e validação de campo. Para classificação dos usos foram divididos em sete classes e, para cada classe foi atribuído um valor de $\mathrm{C}$ conforme encontrado na literatura. Os usos relacionados à água superficial e à área urbana foram considerados como zero, uma vez que nestes locais não há erosão.

Para finalizar, o fator $\mathrm{P}$ foi considerado igual a um, pois não foi considerada nenhuma prática conservacionista em toda a bacia. O mapa de vulnerabilidade à erosão da bacia foi obtido com base nos parâmetros mencionados acima, porém sem considerar a erosividade da chuva, uma vez que não está associado às características da bacia.

A equação da MUSLE foi desenvolvida por Williams (1975) com o intuito de medir a quantidade de sedimentos gerada por um evento de precipitação específico. Neste caso, apenas o fator de erosividade da chuva (fator $\mathrm{R}$ ) foi alterado $\mathrm{e}$ calculado de acordo com a Equação 3.

$$
\mathrm{R}=11,8 *(\mathrm{D} * \mathrm{Qp} * \mathrm{~A})^{0,56}
$$

em que: $D$ se refere ao deflúvio (ou precipitação efetiva), em mm; $Q p$ a vazão de projeto, em m³/s e $A a$ área da bacia hidrográfica, em ha.

A precipitação efetiva foi calculada com base no método da Curva Número $(\mathrm{CN})$, desenvolvido pelo Serviço de Conservação de Solos do Departamento de Agricultura dos Estados Unidos (SCS, 1986), o qual depende do grupo que o solo pertence, ao uso do solo e à umidade antecedente. A equação geral é dada pela Equação 4.

$$
\mathrm{D}=\frac{(\mathrm{Pi}-\mathrm{Ia})^{2}}{\mathrm{Pi}-\mathrm{Ia}+\mathrm{S}}
$$

em que: $D$ é o deflúvio, em mm; Pié a precipitação total, em mm; Ia é a abstração inicial da chuva, correspondente a $20 \%$ de $S$, em $\mathrm{mm}$; e $S$ é o potencial de armazenamento de água no solo, em mm (Equação 5).

$$
\mathrm{S}=\frac{25400}{\mathrm{CN}}-254
$$


A vazão de projeto foi determinada com base no método do Hidrograma Unitário Triangular (HUT), proposto por SCS-USDA, 1986. Segundo Mello e Silva (2013), este método associa os parâmetros da hidrógrafa, vazão de pico e tempo de pico, às características físicas da bacia hidrográfica.

Por fim, foi calculado a taxa de entrega de sedimentos (SDR), que segundo Hamel et al. (2016), se refere ao volume de sedimentos que está sendo perdido pela bacia em relação ao total de sedimentos gerado pela mesma, geralmente expresso em porcentagem (Equação 6).

$$
\operatorname{SDR}(\%)=\left(\frac{\mathrm{A}_{\text {MUSLE }}}{\mathrm{A}_{\text {RUSL E }}}\right) * 100
$$

em que: a $A_{M U S L E}$ se refere à perda de solo transportada para fora da bacia, calculado pela MUSLE e a $A_{\text {RUSLE }}$ diz respeito à perda de solo gerada dentro da bacia, obtida por meio da RUSLE, ambas em t.ha ${ }^{-1} \cdot$ ano $^{-1}$.

\section{RESULTADOS}

\subsection{Erosividade}

Para o ano de 2016 o total precipitado foi aproximadamente $1093 \mathrm{~mm}$, ficando abaixo da média anual para a região de Lavras observada por Curi et al. (2015). A erosividade anual para a bacia em estudo foi de 4745,29 MJ.mm.ha ${ }^{-1} \cdot$ ano $^{-1}$. De acordo com as classes apresentadas por Silva (2004), a precipitação anual incidente na bacia pode ser classificada como média erosividade.

\subsection{Fator $\mathrm{K}$}

Na Figura 2 está apresentada a distribuição espacial dos solos da bacia já com os valores de erodibilidade atribuídos, sendo o valor médio, ponderado pela área, de 0.0234 t.h. $\mathrm{MJ}^{-1} \cdot \mathrm{mm}^{-1}$. Segundo Pereira e Lombardi Neto (2004) e tomando como parâmetro apenas a erodibilidade da bacia, pode-se dizer que apresenta susceptibilidade moderada à erosão.

Figura 2. Distribuição espacial da erodibilidade na área de estudo

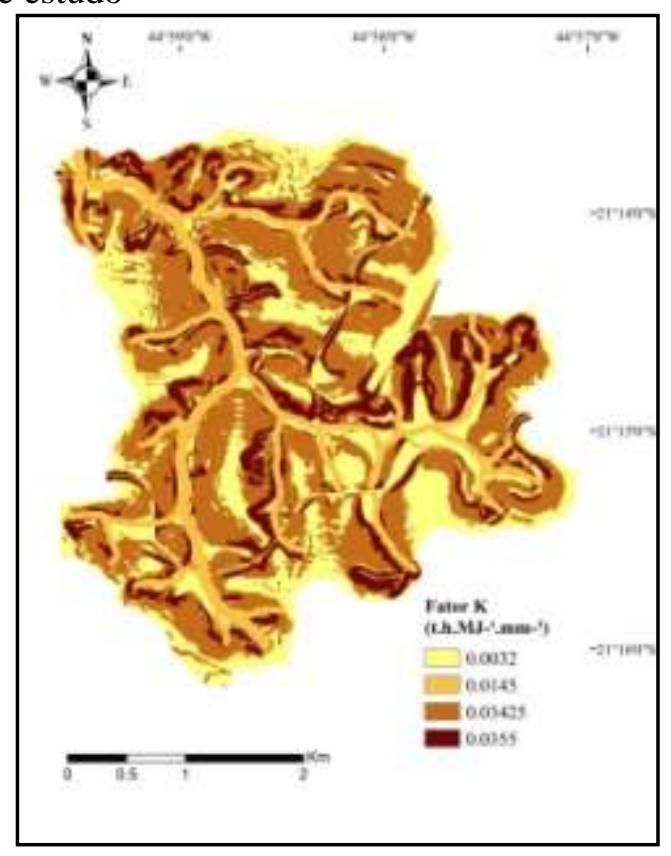

\subsection{Fator LS}

O mapa contendo os intervalos de valores do Fator LS está apresentado na Figura 3 os quais estão compreendidos entre 0 e 30,5 e valor médio ponderado pela área de 3,03. Nota-se que a maior parte da bacia (80\%) apresentou Fator LS igual ou inferior a 5. A declividade da bacia apresentou estreita relação com o Fator LS, onde os maiores valores de declividade resultaram em LS mais elevados, sendo a declividade média 13,52\%. 
Figura 3. Mapa do Fator topográfico para a área de estudo

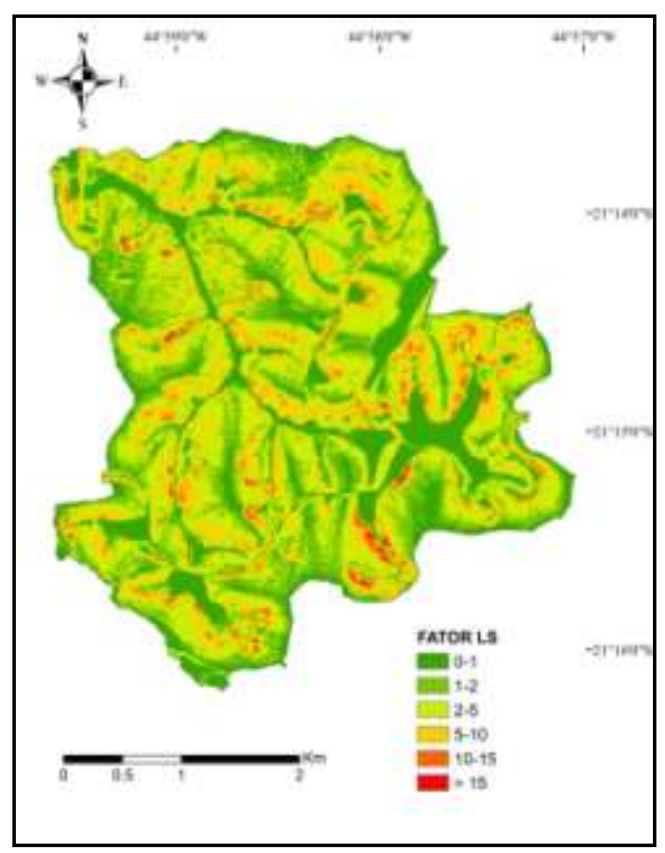

\subsection{Fator C}

O mapa do Fator $\mathrm{C}$ encontrado a partir do uso do solo obtido por meio da classificação supervisionada está apresentado na Figura 3. O uso mais representativo foi de pastagem com 34\%, seguido por área urbana com 27\%, cobertura florestal com aproximadamente 20\% (Mata nativa: $16,22 \%$, e Eucalipto: $3,70 \%$ ) e solo exposto com $10 \%$. Essa parcela de solo exposto aumenta consideravelmente a susceptibilidade à erosão. $\mathrm{O}$ mapa contendo o intervalo de valor do Fator C está apresentado na Figura 4, o qual compreende entre 0 e 0,404 .
Figura 4. Mapa do Fator cobertura para a área de estudo

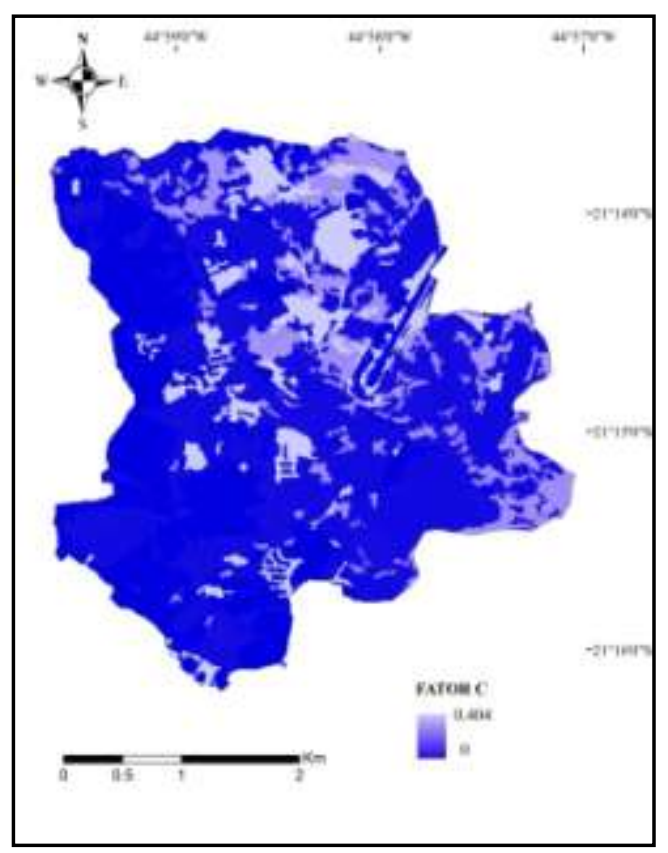

\subsection{Equação Universal de Perda de Solo \\ Revisada - RUSLE}

Foi encontrada a taxa anual de perda de solo para 2016 (Figura 6) correspondente a 37,54 ton.ha ${ }^{-1}$. As áreas mais vulneráveis ao processo erosivo resultaram de regiões que apresentaram maiores declividades associadas com solo exposto e eucalipto. Em contrapartida regiões que apresentaram baixa declividade e com presença de Latossolo, mesmo o solo estando exposto apresentaram baixa susceptibilidade. 
Figura 6. Mapa da perda de solo utilizando a RUSLE

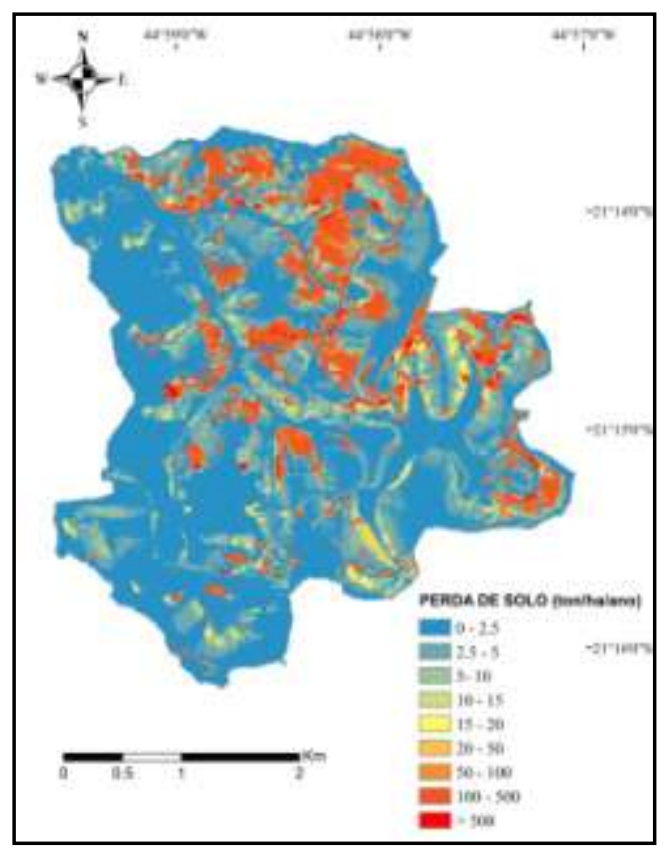

\subsection{Equação Universal de Perda de Solo Modificada - MUSLE}

Foram encontrados 17 eventos erosivos no período de estudo, totalizando uma perda de solo de 0.518 Ton.ha $^{-1}$.ano ${ }^{-1}$. O mês de janeiro foi que apresentou o maior número de eventos bem como a maior perda de solo encontrada no evento do dia 14 com 0.34288 Ton.ha $^{-1}$ correspondente a aproximadamente a $66 \%$ da perda anual na bacia.

\section{DISCUSSÃO}

O índice de erosividade determinado neste trabalho foi muito próximo ao encontrado por Aquino et al. (2012) para o município de Lavras, correspondente a 4865 MJ. mm.ha ${ }^{-1} \cdot$ ano $^{-1}$. Quanto à sazonalidade, nota-se o aumento erosividade com o incremento na quantidade de chuva (OZSOY et al., 2012). Destaques para os meses de janeiro, com a maior precipitação e erosividade, e julho com a ausência de chuva. Silva (2004) estudando a distribuição espacial da erosividade da chuva no Brasil achou uma forte relação deste índice com o total precipitado.

A maior parcela dos solos encontrados é correspondente à classe Argissolo e Cambissolo com pouco mais de $45 \%$ da área, entretanto do ponto de vista da erosão hídrica não seria interessante considerar apenas a erodibilidade, uma vez que dentre os solos da bacia, esta classe é uma das que apresenta maior valor. Estas classes de solos apresentam um horizonte $\mathrm{B}$ incipiente $\mathrm{e}$ devido a sua textura favorece o selamento superficial reduzindo capacidade de infiltração (BESKOW, 2009).

O mapa contendo os intervalos de valores do Fator LS está apresentado na Figura 3 os quais estão compreendidos entre 0 e 30,5 e valor médio ponderado pela área de 3,03. Nota-se que a maior parte da bacia (80\%) apresentou Fator LS igual ou inferior a 5. A declividade da bacia apresentou estreita relação com o Fator LS, onde os maiores valores de declividade resultaram em LS mais elevados, sendo a declividade média 13,52\%.

Na determinação do Fator $\mathrm{C}$ o valor zero foi atribuído para as áreas urbanas, cursos d'água e represas, áreas que cobrem a maior parte da bacia hidrográfica, pois nessas áreas não há erosão e transporte de sedimentos. O maior valor $(0,404)$ foi atribuído para as áreas de solo exposto, que por não possuírem cobertura vegetal não reduzem a energia cinética da precipitação que atinge o solo (RUHOFF et al., 2006). As áreas com agricultura, eucalipto e mata nativa, às quais foram atribuídos valores intermediários, a presença de cobertura vegetal possibilita a redução da energia cinética econsequentemente da susceptibilidade aos processos erosivos. 
O Latossolo apresentou a menor perda de solo com mais de $90 \%$ do material erodido presente na classe de $0-2,5$ ton.ha ${ }^{-1} \cdot$ ano $^{-1}$. As áreas com presença de Cambissolo foram as que mais contribuíram para a perda de solo na bacia, sendo aproximadamente $19 \%$ da perda de solo na faixa de 10 - 20 ton.ha ${ }^{-1} \cdot$ ano $^{-1}$. Além das características intrínsecas destes solos como já discutido, a posição deles na bacia contribuiu para estes valores, pois os Cambissolos foram posicionais em regiões com declividades mais acentuadas.

A quantidade de sedimentos total gerada na bacia de estudo em 2016 foi de 0,518 ton.ha ${ }^{-1}$, valor este próximo ao valor médio encontrado por Beskow et al (2009) para a bacia do Rio Grande também localizada no estado de Minas Gerais, em estudo realizado com os dados de 1996 a 2003.

A partir dos dados encontrados com a RUSLE e MUSLE foi obtida a taxa de entrega de sedimentos anual na bacia hidrográfica de 0,0138, ou seja, estima-se que em 2016 1,38\% do total da carga de sedimento gerada na bacia, por erosão, foi transportada até a seção de controle. Valores estes muito próximos aos valores médios encontrado por Beskow et al (2009) de 0,016 e 1,62\%. Essa diferença, apesar de pequena, pode ser explicada pelo período de determinação do SDR, uma vez que em 2016 a precipitação correspondeu a apenas $75 \%$ da média histórica.

Santos et al. (2017) encontraram taxas de entrega anual de sedimento menores para a região semiárida do nordeste brasileiro, de $0,48 \%$ e $0,26 \%$ para os anos de 2013 e 2014 respectivamente Estes valores inferiores estão provavelmente relacionados com a baixa quantidade de precipitação e as áreas susceptíveis a deposição de sedimentos que por sua vez reduzem a taxa de entrega de sedimentos.

Worral et al. (2014) avaliaram o SDR em 192 bacias hidrográficas localizadas no Reino Unido, com área de drenagem variando entre $4 \mathrm{e}$ $9948 \mathrm{~km}^{2}$, para um período de 36 anos (1974 a 2010). Os autores perceberam uma relação inversa entre a área da bacia e a taxa de entrega de sedimentos, ou seja, para bacias menores, a taxa era consideravelmente maior, o que implica em uma maior deposição de sedimentos nas cabeceiras dos rios. Essa correlação negativa entre a produção de sedimentos e a área de drenagem foi interpretada em função da mudança no uso do solo, da hidrologia e de fatores climáticos.

\section{CONCLUSÕES}

A partir da aplicação da Equação Universal da Perda de Solo Modificada (RUSLE) para a bacia hidrográfica do Ribeirão Vermelho em Lavras-MG foi possível identificar que perda anual de solo para o ano de 2016 foi de a 37,54 ton.ha ${ }^{-1}$. Entretanto, 69\% da bacia hidrográfica apresentou uma taxa anual de perda de solo baixa, de até 5 ton.ha ${ }^{-1}$, que possivelmente está relacionada com a parcela da bacia ocupada por área urbana impermeabilizada.

As áreas mais susceptíveis à erosão foram as com maior declividade associada ao solo exposto e eucalipto. As áreas com presença de Cambissolo também apresentaram maior susceptibilidade, sendo que essas áreas também estão associadas às declividades mais acentuadas. Já as áreas com menor susceptibilidade foram as que apresentaram menor declividade e presença de 
Latossolo, mesmo quando associadas ao solo exposto.

A quantidade de sedimentos total gerada na bacia de estudo no ano de 2016 foi de 0,518 ton.ha ${ }^{-1}$, sendo que $66 \%$ do total gerado no ano de 2016 está associado a um único evento ocorrido na estação chuvosa.

A taxa de entrega de sedimento (SDR) anual na bacia hidrográfica foi 0,0138 , ou seja, $1,38 \%$ do total de sedimento gerada na bacia por erosão, foi transportada até a seção de controle, quantidade está muito próxima ao encontrado na literatura para uma bacia hidrográfica também localizada no sul do estado de Minas Gerais.

Neste sentido é possível identificar que a metodologia aplicada se mostrou adequada para atingir os objetivos propostos. E, para o manejo adequado do uso do solo na bacia em questão e consequentemente a redução da perda de solo e da taxa de entrega de sedimentos é necessária a alteração do uso do solo e a inserção de práticas conservacionistas nas áreas mais suscetíveis à erosão, ou seja, as áreas com solo exposto, principalmente às associadas ao Cambissolo e às altas declividades.

\section{REFERÊNCIAS}

AQUINO, R. F. et al. Spatial variability of the rainfall erosivity in southern region of Minas Gerais state, Brazil. Ciência e Agrotecnologia, v. 36, n. 5, p. 533542, 2012. http://dx.doi.org/10.1590/S141370542012000500006

BONILLA, C. A.; REYES, J. L.; MAGRI, A. Water Erosion Prediction Using the Revised Universal Soil Loss Equation ( Rusle ) in a Gis Framework, Central Chile. Chilean Journal of Agricultural Research, v. 70, n. 1, p. 159-169, 2010.

CURI, P. N. et al. Production of blackberry and redberry in Lavras-MG, Brazil. Ciência Rural, v. 45, n. 8, p. 1368-1374, 2015. http://dx.doi.org/10.1590/0103$8478 \mathrm{cr} 20131572$
DELGADO, M. I.; GASPARI, F. J.; KRUSE, E. E. Land Use Changes and Sediment Yield on a Hilly Watershed in Central-East Argentina.

Soil\&WaterResearch, v. 10, n. 3, p. 189-197, 2015. https://doi.org/10.17221/49/2014-SWR

DISCONZI, et al., Erodibilidade do Solo da Bacia Da Barragem Santa Bárbara, Pelotas-RS.

SociedadeBrasileira de Ciência do Solo. 2014.

FLANAGAN, D. C.; GILLEY, J. E.; FRANTI, T. G. Water Erosion Prediction Project (WEPP):

Development History, Model Capabilities, and Future Enhancements. Biological Systems Engineering:

Papers and Publications, v. 50, n. 5, p. 1603-1612, 2007. https://doi.org/10.13031/2013.23968

GRAVES, A. R. et al. The total costs of soil degradation in England and Wales. Ecological Economics, v. 119, p. 399-413, 2015.

https://doi.org/10.1016/j.ecolecon.2015.07.026

HAMEL, P. et al. Sediment delivery modeling in practice: Comparing the effects of watershed characteristics and data resolution across hydroclimatic regions. Science of the Total Environment, v. 580, p. 1381-1388, 2017.

https://doi.org/10.1016/j.scitotenv.2016.12.103

KAYET, N. et al. Evaluation of soil loss estimation using RUSLE model and SCS-CN method in hilltop mining areas. International Soil and Water Conservation Research, 2018. https://doi.org/10.1016/j.iswcr.2017.11.002

KHATUN, S. Detection of Soil Erosion Potential Zones and Estimation of Soil Loss in Kushkarani River Basin of Eastern India. Journal of Geography, Environment and Earth Science International. v. 12, n. 4, p. 1-17. 2017. https://doi.org/10.9734/JGEESI/2017/37296

KINNELL, P. I. A. Event soil loss, runoff and the Universal Soil Loss Equation family of models : A review. Journal of Hydrology v. 385, p. 384-397, 2010. https://doi.org/10.1016/j.jhydrol.2010.01.024

KINNELL, P.I.A., RISSE, L.M. USLE-M: empirical modeling rainfall erosion through runoff and sediment concentration. Soil Science Society of America Journal, v. 62, p. 1667-1672, 1998. https://doi.org/10.2136/sssaj1998.036159950062000600 $26 \mathrm{x}$

MARQUES, J. D., ALVARENGA, R. C., CURI, N., SANTANA, D. P., SILVA, M. L. Índices de erosividade da chuva, perdas de solo e fator erodibilidade para dois solos da região dos cerrados: primeira aproximação. Revista Brasileira de Ciência do Solo. v. 21, p. 427-434 1997.http://dx.doi.org/10.1590/S010006831997000300011 
MARTINS, S. G. et al. Fator cobertura e manejo do solo e perdas de solo e água em cultivo de eucalipto e em Mata Atlântica nos Tabuleiros Costeiros do estado do Espírito Santo. ScientiaForestalis, v. 38, n. 87, p. 517 $526,2010$.

OLIVEIRA, A. M. M., PINTO, S. A. F., LOMBARDI NETO, F. Caracterização de indicadores da erosão do solo em bacias hidrográficas com o suporte de Geotecnologias e modelo preditivo. Estudos Geográficos: Revista Eletrônica de Geografia. v. 5, n. 1, p. 63-86. 2007.

OZSOY, G. et al. Determination of soil erosion risk in the mustafakemalpasa river basin, Turkey, using the revised universal soil loss equation, geographic information system, and remote sensing.

Environmental Management, v. 50, n. 4, p. 679-694, 2012. https://doi-org /10.1007/s00267-012-9904-8

PAN, J.; WEN, Y. Estimation of soil erosion using RUSLE in Caijiamiao watershed, China. Natural Hazards, v. 71, n. 3, p. 2187-2205, 2014. https://doi-org /10.1007/s11069-013-1006-2

RENARD, K. G. et al. RUSLE: Revised universal soil loss equation. Journal of Soil and Water Conservation, v. 46, n. 1, p. 30-33, 1991.

RICKSON, R. J. et al. Input constraints to food production : the impact of soil degradation. Food Security, v. 7, p. 351-364, 2015. https://doi-org /10.1007/s12571-015-0437-x

RUHOFF, A. L. et al. Avaliação dos processos erosivos através da equação universal de perdas de solos, implementada com algoritmos em legal. Geomática. v. 1, n. 1, p. 12-22, 2006.

SANTOS, J. C. N. et al. Determinação do fator de cobertura e dos coeficientes da MUSLE em microbacias no semiárido brasileiro. Revista Brasileira de Engenharia Agrícola e Ambiental, v. 18, n. 11, p. 1157-1164, 2014. https://doi.org/10.1590/18071929/agriambi.v18n11p1157-1164

SILVA, A. M., Erosividade da Chuva e Erodibilidade de Cambissolo e Latossolo na Região de Lavras, sul de Minas Gerais. Revista Brasileira de Ciência do Solo. v. 33, p. 1811-1820, 2009.

USDA, S. C. S. Urbanhydrology for smallwatersheds. Technical release, v. 55, p. 2-6, 1986.

WILLIAMS, J. R. Sediment-yield prediction with universal equation using runoff energy factor. In Present and Prospective Technology for Predicting Sediment Yields and Sources, Proceedings of the SedimentYield Workshop, USDA Sedimentation Laboratory, Oxford, Mississippi, November 28-30, pp.244-252. 1975.

WISCHMEIER, W.C., SMITH, D.D. Predicting Rainfall Erosion Losses - A Guide to Conservation Planning. Agricultural Handbook, n. 537, 1978.

WISCHMEIER, W.C., SMITH, D.D. Predicting Rainfall Erosion Losses From Cropland East of the Rocky Mountains. Agricultural Handbook, n. 282, 1965.

WORRALL, F. et al. Variation in suspended sediment yield across the UK-A failure of the concept and interpretation of the sediment delivery ratio. Journal of hydrology, v. 519, p. 1985-1996,

2014.https://doi.org/10.1016/j.jhydrol.2014.09.066

ZHANG, H. et al. Extension of a GIS procedure for calculating the RUSLE equation LS factor. Computers \& Geosciences, v. 52, p. 177-188, 2013.

Vanessa Alves Mantovani
Doutoranda em Recursos Hídricos em Sistemas Agrícolas,
UFLA.

\section{Jhones da Silva Amorim}

Doutorando em Recursos Hídricos em Sistemas Agrícolas, UFLA.

André Ferreira Rodrigues
Mestrando em Recursos Hídricos em Sistemas Agrícolas,
UFLA.

Rubens Junqueira
Mestrando em Recursos Hídricos em Sistemas Agrícolas,
UFLA.

Ronan Naves Carvalho
Doutorando em Recursos Hídricos em Sistemas Agrícolas,
UFLA.

\begin{tabular}{l} 
Alisson Souza de Oliveira \\
Professor Doutor do Curso de Mestrado em \\
Sustentabilidade em Recursos Hídricos - UninCor. \\
\hline
\end{tabular}

